\title{
Peripheral Inserted Central Catheter Use and Related Infections in Clinical Practice: A Literature Update
}

\author{
Dimitrios Velissaris $^{\mathrm{a}, \mathrm{f}}$, Vasileios Karamouzos ${ }^{\mathrm{b}}$, Maria Lagadinou ${ }^{\mathrm{c}}$, \\ Charalampos Pierrakos ${ }^{\mathrm{d}}$, Markos Marangos ${ }^{\mathrm{e}}$
}

\begin{abstract}
Peripherally inserted central catheter (PICC) lines are commonly used by clinicians in daily practice. This strategy has been established the latest years as a common approach in many clinical conditions. Apart from their usefulness, PICC use is related to some complications. Aim of this review is to summarize all relevant publications regarding the PICC-related infection, as sepsis remains a high mortality syndrome. We conducted a PubMed search to identify all relevant publications referring to infective complications after insertion and use of PICC lines in hospitalized adult patients. A great number of publications suggest that PICC lines are widely used in the management of patients. The use of peripheral inserted central lines is related with a few complications, including bloodstream infections. Existing data mainly support their use in specific clinical conditions because of the low infectious rates. Some conflicting data also exist regarding PICC use, due to an unclear benefit from their use compared to other commonly used strategies. Although a number of complications, including bloodstream infections are related with insertion of PICC lines, their use has a promising role and can be used when indicated in a wide variety of clinical conditions, especially in specific categories of patients and prolonged periods.
\end{abstract}

Keywords: Peripheral inserted central catheter; Bloodstream infection; Sepsis

\section{Introduction}

Catheterization of central veins constitutes one of the most

Manuscript submitted January 16, 2019, accepted February 18, 2019

anternal Medicine Department, University of Patras, University Hospital of Patras, Greece

bIntensive Care Unit, University Hospital of Patras, Greece

'Emergency Department, University Hospital of Patras, Greece

Intensive Care Department, Brugmann University Hospital, Brussels, Belgium

eDepartment of Infectious Diseases, University of Patras, Greece, University Hospital of Patras, Greece

${ }^{f}$ Corresponding Author: Dimitrios Velissaris, Internal Medicine Department, University of Patras, University Hospital of Patras, Greece.

Email: dvelissaris@upatras.gr

doi: https://doi.org/10.14740/jocmr3757 common procedures in daily clinical practice either for monitoring of central venous pressures or for administration of fluids, medications, chemotherapy and parenteral nutrition for prolonged period. Peripherally inserted central catheters (PICCs) are a worldwide used central intravenous (IV) access device, which has replaced partially central inserted lines as part of daily clinical practice especially in cases with concrete indications [1-3]. A number of complications are related to the use of PICC lines. These include redness at insertion site or discomfort, also some restriction in daily activities and difficulties with flushing or operating the device. However, severe complications can also develop such as life-threatening bloodstream infection and deep vein thrombosis. Sepsis remains a major problem worldwide with high morbidity and mortality rates and the presence of a PICC line device has been shown to contribute to increased rates of related bloodstream infections. Herein, we present a report regarding the influence of sepsis due to the use of PICCs. A review of the literature was conducted for that purpose.

\section{Literature Search}

We conducted a PubMed search on December 2018 using the terms "peripheral inserted central catheters and infections" as "Title/Abstract" or as "MeSH terms". The structure of the search in the "Search details" window of the PubMed website was "peripheral" (All Fields) AND "inserted" (All Fields) AND "central" (All Fields)) AND ("catheters" (MeSH terms) OR "catheters" (All Fields) OR "catheter" (All Fields)) AND ("infection" (MeSH Terms) OR "infection" (All Fields) OR "infections" (All Fields)). Only articles referring to adult humans were enrolled in this review. Bibliographies of the extracted manuscripts were further searched for additional relevant publications. We enrolled studies that referred only to hospitalized patients, either in intensive care unit (ICU) setting or general hospital ward. PubMed search revealed finally 378 publications. In this review we enrolled studies which were focused only to infections related to the insertion and use of PICC lines in hospitalized patients. We excluded all publications who referred to neonates and children, and the ones who referred to any mechanical complications or related to quality of life in the out-of-hospital setting. Case reports and publications written in language other than English were excluded. Finally, 40 studies were found eligible for inclusion in this review, as shown in Figure 1. 


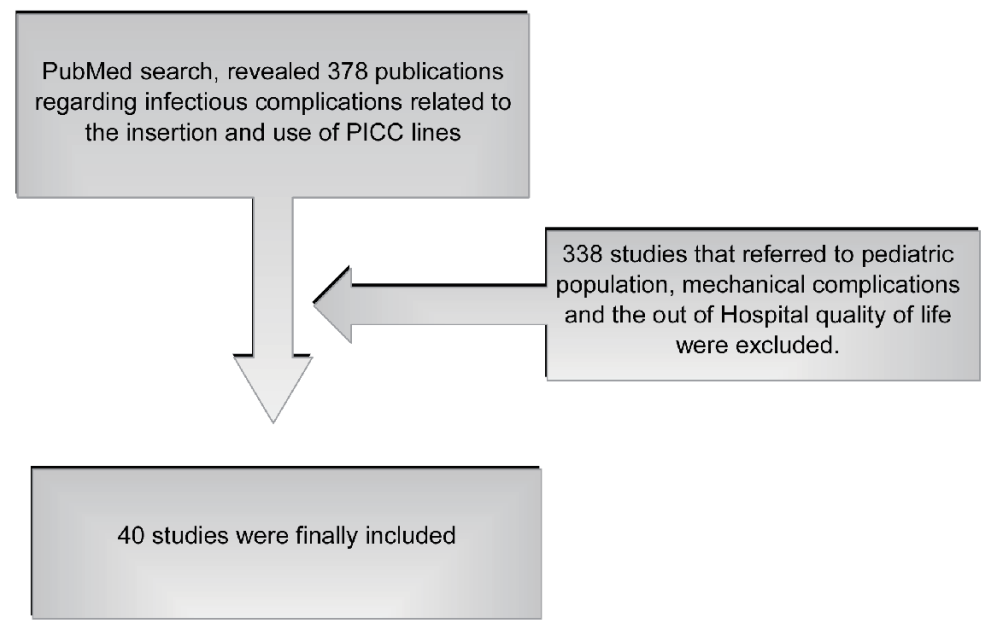

Figure 1. Literature search method.

\section{Literature Retrieved}

A retrospective study from a single center in Brazil analyzed 1,057 medical records. A total of 720 PICCs were inserted at this Institute of Orthopedics and Traumatology over a 10-years period. No cases of PICC line infections were documented after culture of samples from catheter tips and peripheral blood. The authors concluded that this low infectious rate was probably associated with the less severe clinical condition of orthopedic patients and maybe the use of smaller caliber PICC with a single lumen [4].

A multi-center retrospective study from Korean Hospitals published in 2018, aimed to evaluate the impact of subcutaneous tunneling on PICC placement in terms of central lineassociated bloodstream infections (CLABSIs). The results of the study showed a reduction rate of CLABSI related to a subcutaneous tunneling approach for PICC placement. Other risk factors (age, gender, comorbidity, PICC duration, hospital and ICU stay) showed no significant correlations with CLABSI [5].

A retrospective study by Kagan et al, also in 2018, when assessing the risk factors for CLABSI, by comparing the antimicrobial-impregnated peripherally (AIP) central catheters versus the non-antimicrobial-impregnated (NAIP) catheters, found that CLABSIs were higher among patients receiving NAIP catheters. The authors concluded that higher risk for CLABSI was associated with placement of a tunneled catheter, AIDS patients, leukemia, and if the indication for PICC placement was chemotherapy [6].

A retrospective study by Stewart enrolled 357 patients with Staphylococcus aureus bacteremia (SAB) compared safety of early vs. late PICC line insertion. The insertion was defined as early when the catheter was inserted within $48 \mathrm{~h}$ after a positive blood culture. There were not any significant differences between the early and late PICC insertion groups regarding mortality, duration of bacteremia and relapsed SAB. No confirmed PICC infections were identified in either group. They concluded that early PICC line insertion in SAB appears to be safe [7].

Using data from the Michigan Hospital Medicine Safety consortium, a study was conducted enrolling patients that experienced PICC-CLABSI. Of 23,088 patients who received PICCs during the study period (January 2013 to October 2016), $1.1 \%$ developed CLABSI. Significant risk factors associated with PICC-CLABSI included hematological cancer, CLABSI within 3 months of PICC insertion, multi-lumen PICC, solid cancers with ongoing chemotherapy, total parenteral nutrition applied through the PICC, and presence of another central venous catheter (CVC) at the time of PICC placement. The Michigan PICC-CLABSI (MPC) score was significantly associated with risk of CLABSI $(\mathrm{P}<0.0001)$. The study concluded that the MPC score offers a novel way to inform decisions regarding PICC use, surveillance of high-risk cohorts, and utility of blood cultures when PICC-CLABSI is suspected [8].

A retrospective review of all PICCs placed in the ICU setting at an institution during a period of 1 year was evaluated by Martyak et al. Two groups of patients were studied: those with the PICCs placed at the bedside in the ICU and those placed by interventional radiology in non-ICU patients. Results showed that PICC lines placed at the bedside in the ICU setting were associated with higher complication rates, particularly infective, than those placed by interventional radiology in the nonICU setting. Based on these results, the authors concluded that routine placement of PICC lines in the ICU setting needs reevaluation [9].

Prospectively data from an academic tertiary center in Switzerland were collected in a surveillance study evaluating both infectious and noninfectious outcomes. A total of 135 PICCs were inserted in 124 mainly oncology patients. The overall rate of complications was 4.5 per 1000 catheter-days. Of great importance was the successful introduction of PICCs in the academic hospital, by implementing a systematic surveillance program for complications. Both infectious and noninfectious complications were rare [10].

A publication in 2017 referred to the safety and efficacy of a novel non-antibiotic catheter lock solution for the prevention of CLABSI. The solution was consisted of 15 or $30 \mu \mathrm{g} / \mathrm{mL}$ of 
nitroglycerin combined to $4 \%$ sodium citrate and $22 \%$ ethanol. Sixty patients with hematologic malignancies with PICC were enrolled in order to receive the study lock solution. Each lumen of the PICC was locked for at least $2 \mathrm{~h}$ once daily prior being flushed. Results of the study showed that prophylaxis is safe, well tolerated and may prevent CLABSI [11].

The incidence of complications in pregnant and postpartum patients after PICC insertion was reported in a retrospective case series. Totally 112 patients were enrolled in the study and infection rate was $3.6 \%$. In total, the complication rates in this population were similar to that in non-pregnant population [12].

A prospective, multi-center, cohort study of cancer patients with PICC insertion published in 2017 enrolled 477 for a total of 50,841 catheter days. Seventeen percent of the patients developed PICC-line complications and CLABSI was reported in $1.3 \%$ of them. In regard to other factors, patients with body mass index (BMI) greater than 25 were more likely to have PICC complications [13].

The feasibility and safety of PICC for use in acute myeloid leukemia patients was recorded in 89 patients. The PICC increased the quality of life in these patients during chemotherapy period. Bacteremia in patients with PICCs was comparable to that of other IV lines, suggesting the usefulness of the PICCs [14].

$\mathrm{Xu}$ et al compared the use of PICCs with midline (MC) peripheral catheters. In a retrospective study, a total of 206 PICCs and $200 \mathrm{MCs}$ were inserted in 367 patients, and the results showed that MCs were associated with a higher risk of non-life-threatening complications versus PICCs, which showed fewer but more serious complications, including bacteremia [15].

Quality and cost measurements for 200 PICCs post-institution of a mandatory electronic communication tool (MECT), based on clinical practice guidelines were compared with 200 PICCs 12 months prior. Among other results, significant outcomes included a decrease rate in central-line associated bloodstream infection after application of MECT [16].

The impact of chlorhexidine (CHG)-impregnated versus non-CHG PICCs on risk of CLABSI was evaluated in the study by Storey in 2016. After analyzing data from 167 patients, it was shown that no significant difference was noted in the development of CLABSI in the two groups of patients [17].

Results from a retrospective review of prospectively collected data after the creation of a dedicated surgical PICC team who aimed to reduce the complications of PICCs placement were published in 2016. For the period between 2000 and 2013, 35,651 PICC placements were requested, but 24,638 (69.1\%) were finally approved. Ninety-five percent of the PICCs were placed at the bedside within 1 day of approval. Bloodstream infections occurred in $5.9 \%$ of cases. It was showed that the implementation of a surgeon-led PICC team had a significant impact on the placement rate, reducing complications [18].

A prospective study from Bertoglio investigated the complications of the PICC insertion in oncology patients when receiving chemotherapy. PICC was considered a safe approach in the 291 enrolled patients, as among other complications with low incidence, CLABSI had a rate of 0.95 per 1,000 catheter days [19].
A retrospective cohort study based on data in an adult medical ICU at Mayo Rochester of 200 PICCs (dual/triple lumen) and 200 centrally inserted central catheter CICCs (triple/ quadruple lumen) was conducted to compare the complications of both groups. Complications such as thrombosis and infections were uncommon following PICC and CICC insertion, with no significant difference in complication rates [20].

A retrospective, single center cohort analysis of oncology patients aimed to compare the incidence of thrombosis and infections in two groups who had either peripherally-inserted (PICCs) or long-term skin tunneled catheters (LTSTCs). In regard to infection, incidence rate was higher in the PICC group [21].

A retrospective observational study published in 2015, aimed to assess patients with CLABSI in the non-ICU setting. Over a 2-year period, 113 CLABSIs were recorded in 104 patients, with an infection rate of $0.35 / 1,000$ patient days. The study concluded that in non-ICU patients CLABSI incidence was higher in patients with neutropenia, hematologic malignancy, and PICC lines [22].

In 2015 a systematic review of prospective and retrospective studies in the English language for the period January 2000 to October 2013 was published aiming to identify the post-insertion PICC complication rates in adults. The study was especially focused in the complications difference between silicone and polyurethane lines. Overall, PICC complication rates ranged from 8-50\%. Both types of PICCs exhibit nearly identical post-insertion compilation rates. Oncology patients experienced increased post-insertion complications [23].

Austin et al conducted a study aiming to compare PICCassociated complication rates in both the critical care and burn unit. This retrospective cohort review referred to 53 patients with a total of 73 PICC lines in a regional burn unit for a 5 -years period. PICC line-associated complication rates including infections were similar to those published in the critical care literature. The authors concluded that PICC lines can be a useful tool for the treatment of burn patient, but their use are related to significant and potentially fatal risks [24].

An observational study referring to CVC lines insertion in 55 patients with solid tumors for required IV chemotherapy was performed. Data were recorded from patients with tunneled cuffed silicone catheters, PICC lines and central venous ports, aiming to compare the complication and infectious rates. The results showed that central venous ports and PICC lines in this case series of oncology patients had lower line infection rates than tunneled catheters [25].

A retrospective review was performed in patients with a PICC admitted to a regional burn center in US from 2006 to 2008. Fifty-six patients received a PICC line and eight developed a PICC infection with an overall rate of 11.7/1,000 catheter days. Patients with severe burn injury, long hospital stay, and delayed PICC placement during hospitalization had a higher risk of developing PICC infection [26].

Significant reduction in PICC-related infections and thrombosis were reported as a result of a program implementation in a university center in Canada. This program was based on the evaluation of the necessity of the number of lumen of PICC line (two lumens vs. one) and the inappropriate catheter care on the wards. Finally minimum number of lumens 
reduces complications and costs [27].

A meta-analysis by Chopra et al in 2013 was designed to compare the risk of CLABSI between PICCs and CVCs. After extracting relevant publications from medical databases, 23 studies involving 57,250 patients met the eligibility criteria. Pooled meta-analyses of the studies showed that PICC-related CLABSI occurred as frequently as CLABSI from CVCs. It was finally concluded that although PICCs are associated with a lower risk of CLABSI than CVCs in outpatients, hospitalized patients may be just as likely to experience CLABSI with PICCs as with CVCs [28].

A prospective study published in 2013, was based on data from 267 PICCs inserted in 222 patients for several reasons in interventional radiology. Results showed 20 infectious complications $(10 \%)$, which led to removal of the PICC. The authors mentioned the usefulness of this alternative to central lines, also the fact that complications rate was high [29].

A retrospective cohort study from a large referral hospital in San Francisco aimed to assess the relation between CLABSI or venous thrombus (VT) and PICC adjustment due to tip malposition. Fifty-seven CLABSIs were identified in the studied population. Immunosuppression and three PICC lumens were associated with increased risk of CLABSIs; also power-injectable PICCs were associated with increased risk of CLABSI and VT formation. Post-placement adjustment of PICCs was not associated with increased risk of CLABSI or VT [30].

A retrospective study by Armstrong examined the impact of antibiotic impregnated PICCs on the bacteremia rate in a regional burn center. Nineteen patients were enrolled over a 2 -year period and it was concluded that bacteremia rate for the study group was $0 \%$ compared to a $50 \%$ rate of the retrospective control group [31].

A review published in 2012 investigated the incidence of central and peripheral venous catheter-related bloodstream infections in critically ill surgical patient. The study retrieved eight articles published in the period from 1999 to 2011. Due to the diverse definitions for the diagnosis of central and peripheral venous bloodstream infection (BSI) along with the vastly different sample size and extremely small PICC population size at that time, no safe conclusions could be drawn [32].

A review article regarding the reduction of bloodstream infections during catheter insertion was published in 2012 by Petree. Except sterilization of the area of PICC insertion, needleless connectors, positive-pressure valves, and proper securement with self-adhesive anchoring devices were found to be more effective [33].

The rate of the of PICC-associated bloodstream infections was reported in a prospective cohort study from a hospital in Saudi Arabia. Ninety-two PICC lines were inserted with a total of 3,336 device-days. The overall BSI rate was 4.5/1000 PICC days. This study suggested that underlying conditions and indications for the PICC line use may play an important role in the BSI occurrence [34].

A prospective cohort study which assessed the CVC bloodstream infections in cancer patients compared to other approaches including PICCs, concluded that PICCs had a lower infection rate. This study provided further support for the use of PICC line in hematology-oncology population [35].

A retrospective review was performed for all chronic he- modialysis catheter placements and exchanges at a large university hospital for a 5-year period. Results showed that $20.5 \%$ of patients had a history of PICC placement, and these patients were more likely to have catheter-related infections compared to patients without a history of PICC placement [36].

In a non-randomized study referring to surgical ICU patients with a prolonged stay, PICC was associated with fewer related infections, although CVCs were in place longer than PICCs. The infection rate was 6.0/1,000 catheter-days for CVCs and 2.2/1,000 for PICC lines [37].

A review study by Fearonce et al aimed to compare the use and safety of PICCs vs. CVCs in burn patients who were treated in a single US center who received one or more PICCs during a 2-year period. PICCs seem to be more effective and safer as bloodstream infectious rate for PICCs was 0 per 1,000 line-days, whereas for CVCs it was 6.6 per 1,000 line-days [38].

A prospective study sought to assess patients with CVCs in the non-ICUs setting and those with PICCs hospital-wide. Results showed that CLABSI had a rate of 2.4 per 1,000 catheter-days and for the PICCs the rate was 2.3 per 1,000 catheterdays. Also, median time to infection onset was significantly longer in patients with a PICC ( 23 vs. 13 days; $\mathrm{P}=0.03$ ). This longer period suggest PICCs as a safer approach for prolonged IV access [39].

Prospectively collected data regarding complications after insertion of PICCs in oncology patients after implementation of a new placement strategy were reported in the study by Yap et al in 2006. The complication rate including PICC-related infections was significantly lower when compared to data before the introduction of the new strategy [40].

A total of 177 patients were enrolled in a study, aimed to determine any significant differences between the PICCs in relation to the CVC and the peripheral venous access device in respect of the length of stay, incidence of phlebitis and possible removal due to infection. No significant differences were found and the authors concluded that PICCs could be used as an effective alternative [41].

A prospective study by Cowl in 2000 enrolled 102 patients who were receiving total parenteral nutrition (TPN) and compared the complication rates for PICCs and central line insertion. In regard to infection, the overall infection rate was 4.9/1,000 catheter-days and was similar for each catheter type. PICC lines were associated with higher rates of thrombophlebitis [42].

Prospectively collected data from a single institution in Canada were collected for a 10 -year period for patients who received parenteral nutrition. The study was published in 1999 and results showed that PICCs mostly replaced central catheters and their use was not associated with an increased incidence of sepsis [43].

All above are summarized in Table 1 [4-43].

\section{Discussion}

Several medical conditions of various etiologies require the use of a central catheter. PICCs are an established and safe alternative to CVCs inserted into the jugular or subclavian vein, 
Table 1. Literature Retrieved

\begin{tabular}{|c|c|}
\hline Study and year & Study design \\
\hline $\begin{array}{l}\text { Santolim et } \\
\text { al, 2018, [4] }\end{array}$ & $\begin{array}{l}\text { Retrospective study } \\
\text { from a single-center }\end{array}$ \\
\hline $\begin{array}{l}\text { Kim et al, } \\
2018,[5]\end{array}$ & $\begin{array}{l}\text { Multi-center } \\
\text { retrospective study }\end{array}$ \\
\hline $\begin{array}{l}\text { Kagan et al, } \\
2018,[6]\end{array}$ & Retrospective study \\
\hline $\begin{array}{l}\text { Stewart et al, } \\
2018,[7]\end{array}$ & Retrospective study \\
\hline $\begin{array}{l}\text { Herc et al, } \\
2017,[8]\end{array}$ & $\begin{array}{l}\text { Study based on data from } \\
\text { the Michigan Hospital } \\
\text { Medicine Safety consortium }\end{array}$ \\
\hline
\end{tabular}

Martyak et $\quad$ Retrospective review
al, 2017, [9]

al, 2017, [9]

Lo Priore et

al, 2017, [10]

Chaftari et al,

2017, [11]

ive single

institution study

Jacques et al, Retrospective case series

2018, [12]

Kang et al,

2017, [13]

Prospective, multi-

center, cohort study

Chen et al,

2017, [14]

$\mathrm{Xu}$ et al,

2016, [15]

Retrospective study

Retrospective study

Kim-Saechao, Historical cohort study

et al, 2016, [16]

Storey et al,

2016, [17]

Pernar et al,

2016, [18]

Bertoglio et

al, 2016, [19]

Nolan et al, 2016, [20]

\section{Type of patients/ database \\ Major findings}

Patients from

Orthopedics and

Traumatology

Department

Adult patients

with tunneled or conventional PICCs

Adult ill population

Patients with

Staphylococcus aureus bacteremia

Adult patients

After insertion of 720 PICCs, no cases of PICC

line infections were documented after culture of samples from catheter tips and peripheral blood.

Reduction rate of CLABSI related to subcutaneous tunneling approach for PICC placement

PICC CLABSIs were highest among patients receiving non-antimicrobial-impregnated (NAIP) catheters.

Early PICC line insertion in Staphylococcus aureus bacteremia appears to be safe.

The Michigan PICC-CLABSI (MPC) offers a novel way to inform decisions regarding PICC use, surveillance of high-risk cohorts, and utility of blood cultures when PICC-CLABSI is suspected.

Patients with PICCs inserted by the bedside in the ICU and PICCs placed by interventional radiology in non-ICU

Mainly oncology patients

Patients with hematologic malignancies

PICC lines placed at the bedside in the ICU setting were associated with higher complication rates, in particular infectious complications.

Pregnant and postpartum patients

Cancer patients

Acute myeloid leukemia patients

Patients from a large academic medical center

Patients in an academic tertiary medical center

Patients in three high-risk units

Patients' requests for PICCs maintained in database (2000-13)

Decreased infectious rate after implementing a systematic surveillance program

Non-antibiotic catheter lock solution with nitroglycerin, ethanol and sodium citrate reduced CLABSI.

No differences in infectious rate compared to non-pregnant

CLABSI rate $1.3 \%$. Increased MBI was related to more complications.

Bacteremia in patients with PICCs was comparable to that of other IV lines.

PICCs complications were less but more serious (including bacteremia) when compared to midline peripheral catheters

Application of a mandatory electronic communication tool (MECT) based on clinical practice guidelines decreased CLABSI.

No differences in CLABSI development in patients with chlorhexidine (CHG)-impregnated or non-CHG PICC line Implementation of a surgeon-led PICC team had among other, significant impact on the avoidance of complications of PICC lines.

Oncology patients

PICC is a safe venous device for chemotherapy delivery with CLABSI incidence $1.7 \%$.

Adult ITU patients
PICCs (dual/triple lumen) and centrally inserted central catheter CICCs (triple/quadruple lumen) were compared for complications of both groups. Infections were uncommon following PICC and CICC insertion, with no significant difference in complication rates. 
Table 1. Literature Retrieved - (continued)

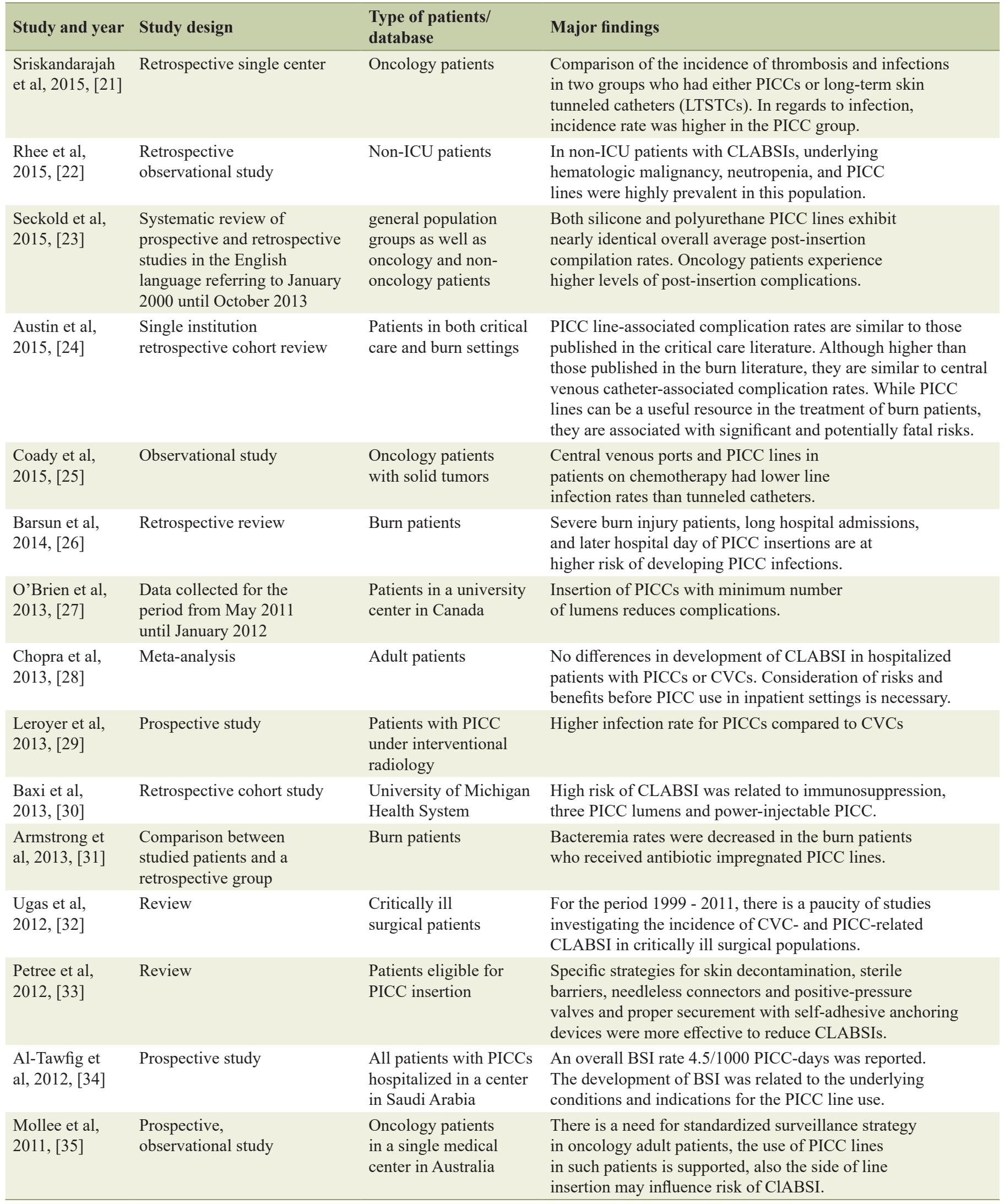


Table 1. Literature Retrieved - (continued)

\begin{tabular}{|c|c|c|c|}
\hline Study and year & Study design & $\begin{array}{l}\text { Type of patients/ } \\
\text { database }\end{array}$ & Major findings \\
\hline $\begin{array}{l}\text { Butler et al, } \\
2011,[36]\end{array}$ & Retrospective review & $\begin{array}{l}\text { Patients in a large } \\
\text { academic hospital }\end{array}$ & $\begin{array}{l}\text { Previous placement of a PICC may be related to catheter- } \\
\text { associated infections in hemodialysis patients. }\end{array}$ \\
\hline $\begin{array}{l}\text { Gunst et al, } \\
2011,[37]\end{array}$ & Non-randomized study & Surgical ICU patients & $\begin{array}{l}\text { PICCs were associated with fewer CLABSIs in long- } \\
\text { stay surgical ICU patient compared to CVCs. }\end{array}$ \\
\hline $\begin{array}{l}\text { Fearonce et } \\
\text { al, 2010, [38] }\end{array}$ & Review & $\begin{array}{l}\text { Burn patients in } \\
\text { a single center }\end{array}$ & $\begin{array}{l}\text { PICC lines had a lower incidence rate for } \\
\text { CLABSI compared to CVCs. }\end{array}$ \\
\hline $\begin{array}{l}\text { Yap et al, } \\
2006,[40]\end{array}$ & $\begin{array}{l}\text { Study based on } \\
\text { prospectively collected } \\
\text { PICC complication data }\end{array}$ & $\begin{array}{l}\text { Oncology patients } \\
\text { with solid tumors }\end{array}$ & $\begin{array}{l}\text { Complication rate for year } 2003 \text { was lower compared to that } \\
\text { of } 2001 \text { probably due to application of related strategies. }\end{array}$ \\
\hline $\begin{array}{l}\text { Cowl et al, } \\
2000,[42]\end{array}$ & Prospective study & $\begin{array}{l}\text { Patients who } \\
\text { received TPN via } \\
\text { a PICC or CVC }\end{array}$ & $\begin{array}{l}\text { Regarding infections, the overall rate was } \\
\text { similar for each catheter type. }\end{array}$ \\
\hline $\begin{array}{l}\text { Duerksen et } \\
\text { al, 1999, [43] }\end{array}$ & $\begin{array}{l}\text { Study based on prospectively } \\
\text { collected data compared over } \\
3 \text { different time periods }\end{array}$ & $\begin{array}{l}\text { Patients who received } \\
\text { parenteral nutrition }\end{array}$ & $\begin{array}{l}\text { Regarding infections, PICCs do not result } \\
\text { in increased line-related sepsis. }\end{array}$ \\
\hline
\end{tabular}

mainly for medium- and long-term intravenous therapy. The use of PICC lines concerns patients with difficult intravenous access, those with specific underlying conditions such as obesity, diabetes or other chronic conditions, oncology patients under chemotherapy treatment and patients receiving total parenteral nutrition. Since the introduction of PICCs in the late 1970s, their use has been increased, especially in the oncology setting, due to their less invasive insertion technique (not requiring implantation or tunneling), the low rate of mechanical complications, and safety with likely lower infectious rates and easy removal techniques [18, 37, 39, 44, 45].

In contrast to these benefits, the safety of PICCs has occasionally been questioned due to the significant number of complications occurring during their use, mostly regarding infections and thrombosis. PICCs are associated with CLABSIs. An infection due to the PICC occurs when bacteria enter the bloodstream through or around the catheter. Also, displaced catheter tips consist of a clinical problem as it can cause central venous thrombosis, vessel wall erosion, also fatal cardiac tamponade. The ideal catheter position has been debated in the literature, but it is generally accepted that the tip should lie within the superior vena cava, adjacent to the atriocaval junction.

The debate among clinicians still exists basically referring to the need for a PICC establishment and the duration of its use. Some strategies have been applied in clinical practice and guidelines for prevention infections due to PICCs. A panel of experts used a validated method to develop appropriate indica- tions for PICC use across patient populations in 2015. After systematic review of the literature, scenarios related to PICC use, care, and maintenance, the international panel developed guidelines according to the type of patient population, the indication of the insertion and the duration of the use of the PICC. They applied the RAND/UCLA Appropriateness Method to develop criteria for use of PICCs. Appropriateness of PICC use was compared to that of other venous access devices. The panel of experts proposed that for peripherally infusions, PICC use was rated as inappropriate when the proposed duration of use was less than 5 days. For use between 6 and 14 days, midline catheters and ultrasonography-guided peripheral IV catheters were preferred to PICCs. Finally, for critically ill patients, non-tunneled CVCs were preferred over PICCs when 14 or fewer days of use were likely [46].

Previously, the ESPEN guidelines published in 2009, addressed infective complications when parenteral nutrition is administered by central lines including PICCs. These complications can be reduced by staff training, hand-washing policies, use of the antiseptic chlorhexidine, appropriate policies for the dressing of the exit site, routine changes of administration sets, and removal of the central lines as soon as they are no longer necessary [47].

Literature review showed that there are specific clinical conditions, such as oncology patients with hematologic malignancies or solid tumors that PICCs are mostly used. Also, surgical patients and these with specific care like burns are candidates for insertion of a PICC line after clinicians' deci- 
sion. It seems that the body literature is growing as there are definitively data which support PICCs use at least in such conditions, but seems to be still a debate in daily clinical practice on whether to insert a PICC line and for how long should this be used. Despite the heterogeneity of the populations in which PICCs have been used, our review identified a number of publications proposing lower infectious rates. Recently, a publication by Kim et al in 2018, proposed that a subcutaneous tunneling approach for PICC insertion significantly reduces the rate of CLABSI [5]. Clinicians working in different medical environments are mostly the ones who are going to make the final decision. For sure, the existence of some other potential complications, basically mechanically and the cost seems to influence this decision.

Some questions still remain to be answered regarding the optimal time of removal of the PICC line, particularly in ICU environment, or the possibility of several PICC insertions over time, also, the indication of PICC insertion in relation to the immune status of the patient as a risk factor for developing sepsis, as well as the indication of PICC insertion in neutropenic patients.

\section{Conclusions}

A great body of literature is referring to the use of PICCs as an alternative option for some specific categories of patients and clinical conditions. Infections related to the insertion of PICC and prolonged use of the catheter are reported, but the risk of bloodstream infections seems not to be higher regarding to classical central catheterization. Applications of specific protocols have eliminated this life-threatening complication. PICC line insertion remains a safe altered option in clinical practice when indicated.

\section{Conflict of Interest}

The authors declare that there is no conflict of interests regarding the publication of this paper.

\section{References}

1. Johansson E, Hammarskjold F, Lundberg D, Arnlind MH. Advantages and disadvantages of peripherally inserted central venous catheters (PICC) compared to other central venous lines: a systematic review of the literature. Acta Oncol. 2013;52(5):886-892.

2. Sainathan S, Hempstead M, Andaz S. A single institution experience of seven hundred consecutively placed peripherally inserted central venous catheters. J Vasc Access. 2014;15(6):498-502.

3. Neuman ML, Murphy BD, Rosen MP. Bedside placement of peripherally inserted central catheters: a cost-effectiveness analysis. Radiology. 1998;206(2):423-428.

4. Santolim TQ, Baptista AM, Giovani AMM, Zumarraga JP, Camargo OP. Peripherally inserted central catheters in orthopedic patients: experience from 1023 procedures.
Acta Ortop Bras. 2018;26(3):206-210.

5. Kim IJ, Shim DJ, Lee JH, Kim ET, Byeon JH, Lee HJ, Cho SG. Impact of subcutaneous tunnels on peripherally inserted catheter placement: a multicenter retrospective study. Eur Radiol. 2018.

6. Kagan E, Salgado CD, Banks AL, Marculescu CE, Cantey JR. Peripherally inserted central catheter-associated bloodstream infection: Risk factors and the role of antibiotic-impregnated catheters for prevention. Am J Infect Control. 2019;47(2):191-195.

7. Stewart JD, Runnegar N. Early use of peripherally inserted central catheters is safe in Staphylococcus aureus bacteraemia. Intern Med J. 2018;48(1):44-49.

8. Herc E, Patel P, Washer LL, Conlon A, Flanders SA, Chopra V. A model to predict central-line-associated bloodstream infection among patients with peripherally inserted central catheters: the MPC score. Infect Control Hosp Epidemiol. 2017;38(10):1155-1166.

9. Martyak M, Kabir I, Britt R. Inpatient Peripherally Inserted Central Venous Catheter Complications: Should Peripherally Inserted Central Catheter Lines Be Placed in the Intensive Care Unit Setting? Am Surg. 2017;83(8):925927.

10. Lo Priore E, Fliedner M, Heverhagen JT, Novak U, Marschall J. The role of a surveillance programme for introducing peripherally inserted central catheters: a 2-year observational study in an academic hospital. Swiss Med Wkly. 2017;147:w14441.

11. Chaftari AM, Hachem R, Szvalb A, Taremi M, Granwehr B, Viola GM, Amin S, et al. A novel nonantibiotic nitroglycerin-based catheter lock solution for prevention of intraluminal central venous catheter infections in cancer patients. Antimicrob Agents Chemother. 2017;61(7):e00091-17.

12. Jacques L, Foeller M, Farez R, Kaljo K, Nugent M, Simpson P, Klatt T. Safety of peripherally inserted central catheters during pregnancy: a retrospective study. J Matern Fetal Neonatal Med. 2018;31(9):1166-1170.

13. Kang J, Chen W, Sun W, Ge R, Li H, Ma E, Su Q, et al. Peripherally inserted central catheter-related complications in cancer patients: a prospective study of over 50,000 catheter days. J Vasc Access. 2017;18(2):153-157.

14. Chen MH, Hwang WL, Chang KH, Chiang LCJ, Teng CLJ. Application of peripherally inserted central catheter in acute myeloid leukaemia patients undergoing induction chemotherapy. Eur J Cancer Care (Engl). 2017;26(6):e12627.

15. Xu T, Kingsley L, DiNucci S, Messer G, Jeong JH, Morgan B, Shutt K, et al. Safety and utilization of peripherally inserted central catheters versus midline catheters at a large academic medical center. Am J Infect Control. 2016;44(12):1458-1461.

16. Kim-Saechao SJ, Almario E, Rubin ZA. A novel infection prevention approach: Leveraging a mandatory electronic communication tool to decrease peripherally inserted central catheter infections, complications, and cost. Am J Infect Control. 2016;44(11):1335-1345.

17. Storey S, Brown J, Foley A, Newkirk E, Powers J, Barger J, Paige K. A comparative evaluation of antimi- 
crobial coated versus nonantimicrobial coated peripherally inserted central catheters on associated outcomes: A randomized controlled trial. Am J Infect Control. 2016;44(6):636-641.

18. Pernar LI, Wolf LL, Seshadri A, Patel V. Impact of a Surgeon-Led Peripherally Inserted Central Venous Catheter Team on Peripherally Inserted Central Venous CatheterRelated Complications and Costs. Surg Infect (Larchmt). 2016;17(3):352-356.

19. Bertoglio S, Faccini B, Lalli L, Cafiero F, Bruzzi P. Peripherally inserted central catheters (PICCs) in cancer patients under chemotherapy: A prospective study on the incidence of complications and overall failures. J Surg Oncol. 2016;113(6):708-714.

20. Nolan ME, Yadav H, Cawcutt KA, Cartin-Ceba R. Complication rates among peripherally inserted central venous catheters and centrally inserted central catheters in the medical intensive care unit. J Crit Care. 2016;31(1):238242.

21. Sriskandarajah P, Webb K, Chisholm D, Raobaikady R, Davis K, Pepper N, Ethell ME, et al. Retrospective cohort analysis comparing the incidence of deep vein thromboses between peripherally-inserted and long-term skin tunneled venous catheters in hemato-oncology patients. Thromb J. 2015;13:21.

22. Rhee Y, Heung M, Chen B, Chenoweth CE. Central lineassociated bloodstream infections in non-ICU inpatient wards: a 2-year analysis. Infect Control Hosp Epidemiol. 2015;36(4):424-430.

23. Seckold T, Walker S, Dwyer T. A comparison of silicone and polyurethane PICC lines and postinsertion complication rates: a systematic review. J Vasc Access. 2015;16(3):167-177.

24. Austin RE, Shahrokhi S, Bolourani S, Jeschke MG. Peripherally inserted central venous catheter safety in burn care: a single-center retrospective cohort review. J Burn Care Res. 2015;36(1):111-117.

25. Coady K, Ali M, Sidloff D, Kenningham RR, Ahmed S. A comparison of infections and complications in central venous catheters in adults with solid tumours. J Vasc Access. 2015;16(1):38-41.

26. Barsun A, Sen S, Palmieri TL, Greenhalgh DG. Peripherally inserted central line catheter infections in burn patients. J Burn Care Res. 2014;35(6):514-517.

27. O'Brien J, Paquet F, Lindsay R, Valenti D. Insertion of PICCs with minimum number of lumens reduces complications and costs. J Am Coll Radiol. 2013;10(11):864868.

28. Chopra V, O'Horo JC, Rogers MA, Maki DG, Safdar N. The risk of bloodstream infection associated with peripherally inserted central catheters compared with central venous catheters in adults: a systematic review and metaanalysis. Infect Control Hosp Epidemiol. 2013;34(9):908918.

29. Leroyer C, Lasheras A, Marie V, Le Bras Y, Carteret T, Dupon M, Rogues AM. Prospective follow-up of complications related to peripherally inserted central catheters. Med Mal Infect. 2013;43(8):350-355.

30. Baxi SM, Shuman EK, Scipione CA, Chen B, Sharma
A, Rasanathan JJ, Chenoweth CE. Impact of postplacement adjustment of peripherally inserted central catheters on the risk of bloodstream infection and venous thrombus formation. Infect Control Hosp Epidemiol. 2013;34(8):785-792.

31. Armstrong SD, Thomas W, Neaman KC, Ford RD, Paulson J. The impact of antibiotic impregnated PICC lines on the incidence of bacteremia in a regional burn center. Burns. 2013;39(4):632-635.

32. Ugas MA, Cho H, Trilling GM, Tahir Z, Raja HF, Ramadan S, Jerjes W, et al. Central and peripheral venous linesassociated blood stream infections in the critically ill surgical patients. Ann Surg Innov Res. 2012;6(1):8.

33. Petree C, Wright DL, Sanders V, Killion JB. Reducing blood stream infections during catheter insertion. Radiol Technol. 2012;83(6):532-540.

34. Al-Tawfiq JA, Abed MS, Memish ZA. Peripherally inserted central catheter bloodstream infection surveillance rates in an acute care setting in Saudi Arabia. Ann Saudi Med. 2012;32(2):169-173.

35. Mollee P, Jones M, Stackelroth J, van Kuilenburg R, Joubert W, Faoagali J, Looke D, et al. Catheter-associated bloodstream infection incidence and risk factors in adults with cancer: a prospective cohort study. J Hosp Infect. 2011;78(1):26-30.

36. Butler PJ, Sood S, Mojibian H, Tal MG. Previous PICC placement may be associated with catheter-related infections in hemodialysis patients. Cardiovasc Intervent Radiol. 2011;34(1):120-123.

37. Gunst M, Matsushima K, Vanek S, Gunst R, Shafi S, Frankel H. Peripherally inserted central catheters may lower the incidence of catheter-related blood stream infections in patients in surgical intensive care units. Surg Infect (Larchmt). 2011;12(4):279-282.

38. Fearonce G, Faraklas I, Saffle JR, Cochran A. Peripherally inserted central venous catheters and central venous catheters in burn patients: a comparative review. J Burn Care Res. 2010;31(1):31-35.

39. Al Raiy B, Fakih MG, Bryan-Nomides N, Hopfner D, Riegel E, Nenninger T, Rey J, et al. Peripherally inserted central venous catheters in the acute care setting: A safe alternative to high-risk short-term central venous catheters. Am J Infect Control. 2010;38(2):149-153.

40. Yap YS, Karapetis C, Lerose S, Iyer S, Koczwara B. Reducing the risk of peripherally inserted central catheter line complications in the oncology setting. Eur J Cancer Care (Engl). 2006;15(4):342-347.

41. Griffiths VR, Philpot P. Peripherally inserted central catheters (PICCs): do they have a role in the care of the critically ill patient? Intensive Crit Care Nurs. 2002;18(1):3747.

42. Cowl CT, Weinstock JV, Al-Jurf A, Ephgrave K, Murray JA, Dillon K. Complications and cost associated with parenteral nutrition delivered to hospitalized patients through either subclavian or peripherally-inserted central catheters. Clin Nutr. 2000;19(4):237-243.

43. Duerksen DR, Papineau N, Siemens J, Yaffe C. Peripherally inserted central catheters for parenteral nutrition: a comparison with centrally inserted catheters. JPEN J Par- 
enter Enteral Nutr. 1999;23(2):85-89.

44. Walker G, Todd A. Nurse-led PICC insertion: is it cost effective? Br J Nurs. 2013;22(19):S9-15.

45. Marschall J, Mermel LA, Fakih M, Hadaway L, Kallen A, O'Grady NP, Pettis AM, et al. Strategies to prevent central line-associated bloodstream infections in acute care hospitals: 2014 update. Infect Control Hosp Epidemiol. 2014;35(Suppl 2):S89-107.

46. Chopra V, Flanders SA, Saint S, Woller SC, O'Grady NP,
Safdar N, Trerotola SO, et al. The Michigan Appropriateness Guide for Intravenous Catheters (MAGIC): results from a multispecialty panel using the RAND/UCLA appropriateness method. Ann Intern Med. 2015;163(6 Suppl):S1-40.

47. Pittiruti M, Hamilton H, Biffi R, MacFie J, Pertkiewicz M, Espen. ESPEN Guidelines on Parenteral Nutrition: central venous catheters (access, care, diagnosis and therapy of complications). Clin Nutr. 2009;28(4):365-377. 